\title{
Applying Output Feedback Integral Sliding Mode Controller to Uncertain Time-Delay Systems with Mismatched Disturbances
}

\author{
Huan-Chan Ting, Jeang-Lin Chang, and Yon-Ping Chen
}

\begin{abstract}
For time-delay systems with mismatched disturbances and uncertainties, this paper develops an integral sliding mode control algorithm using output information only to stabilize the systems. An integral sliding surface is comprised of output signals and an auxiliary full-order compensator. The proposed output feedback sliding mode controller can satisfy the reaching and sliding condition and maintain the system on the sliding surface from the initial moment. When two specific algebraic Riccati inequalities have solutions, our method can guarantee the stability of the closed-loop system and satisfy the property of robust disturbance attenuation. Moreover, the design parameters of controller and compensator can be simultaneously determined by solutions to the algebraic Riccati inequalities. Finally, two numerical examples illustrate the applicability of the proposed scheme.
\end{abstract}

Keywords: Full-order compensator, mismatched disturbance, output feedback, sliding mode.

\section{INTRODUCTION}

Time-delay phenomenon means that parts of system states, inputs, or outputs affect the systems after a fixed time or random but finite period. This phenomenon exists in various practical systems, such as chemical processes, electrical networks, nuclear reactors, biological systems, economic models, etc. Since time delays frequently induce the system instability and bad performance, the analysis and control of time-delay systems, whether state, input, or output delays, have been interesting topics over the past decades. Focusing on the state-delay systems, researchers [1-9] have presented many effective state feedback control methods to various system models. Xia and Jia [1] carried out a robust control method comprising of the sliding mode control and linear matrix inequality (LMI) technique for uncertain time-delay systems with matched disturbances. Lee et al. [6] developed a control method based on the receding horizon concept to stabilize the closed-loop system and to satisfy the $H_{\infty}$ norm bound from the disturbance to the controlled output. For a continuous linear state-delay system involving a class of integral terms, Santos and Mondié [7] proposed an iterative procedure to complete their state feedback controller

Manuscript received January 6, 2010; revised August 12, 2010 and May 11, 2011; accepted June 28, 2011. Recommended by Editorial Board member Poo Gyeon Park under the direction of Editor Young Il Lee.

This work was supported by the National Science Council under grant NSC-100-2221-E-161-003.

Huan-Chan Ting and Yon-Ping Chen are with the Institute of Electrical Control Engineering, National Chiao-Tung University, 1001 University Rd., Hsinchu 300, Taiwan (e-mails: hcting. ece92g@nctu.edu.tw, ypchen@mail.nctu.edu.tw).

Jeang-Lin Chang is with the Department of Electrical Engineering, Oriental Institute of Technology, 58, Sec. 2, Sichuan Rd., Banqiao Dist., New Taipei City 220, Taiwan (e-mail: jlchang@ ee.oit.edu.tw). design. Wang et al. [8] designed a state feedback control law of time-delay systems with system uncertainties and matched unknown nonlinear terms; they combined the LMI technique and adaptive parameter searching law to the controller design ensuring the stability of the closedloop system. Chen and Chen [9] presented an LMI-based state feedback controller and a disturbance observer to stabilize linear state-delay systems with uncertainties and matched disturbances.

When the system states are partly obtainable, both state observers [10-14] and output feedback controllers [15-21] are feasible schemes to stabilize time-delay systems. In the field of state observers, Darouach [14] has developed an observer methodology to estimate states of linear time-delay systems with noises and mismatched disturbances. On the other hand, in the field of output feedback control methods, Fridman and Shaked [15] described explicitly a significant $H_{\infty}$ control method using the descriptor system transformation for time-delay systems with mismatched external disturbances and measurement noises. The descriptor system transformation can simplify the analysis of time-delay systems and perform effectively the disturbance attenuation. Niu et al. [16] extended an observer-based sliding mode control using the LMI technique to regulate uncertain time-delay systems. Yan et al. [19] applied an effective slidingmode design technique using outputs only to control the time-delay systems with disturbances. Pai [21] proposed a Luenberger observer-based output feedback controller for a class of nonlinear uncertain state-delay systems with matched uncertainties and disturbances. This controller was comprised of integral sliding-mode technique and solutions to LMI, which parameters of switching gain are calculated by adaptation laws.

There exists two difficulties in the design of output feedback sliding mode control. The first difficulty is a synthesis problem. Synthesizing a control law using the outputs only is significant since the derivative of the 
sliding surface is always involved with the unmeasured system states. For resolving the synthesis problem, a normal strategy is to add an extra constraint on the controller parameters. The existence of controller parameters is constrained by the extra constraint simultaneously. The other difficulty is to solve an LMI which is necessary, complicated, and with large dimensions for determining the feedback gain stabilizing the closed-loop system. In this paper, an output feedback integral sliding mode controller combining with a fullorder compensator is proposed to improve these two difficulties for time-delay systems with mismatched disturbances and uncertainties. Since mismatched disturbances cannot be eliminated completely even the system is in the sliding mode, the robust disturbance attenuation technique [22-24] can reduce the effect of disturbances acting on a system to an acceptable level. $H_{\infty}$ robust control method for the disturbance attenuation is a successful strategy to minimize the gain from external disturbances to the controlled output over all frequencies. Introducing the $H_{\infty}$ robust control method into the proposed controller can guarantee the robust stability of the closed-loop system and accomplish the property of robust disturbance attenuation. Moreover, the solutions to two algebraic Riccati inequalities determine the design parameters of the integral sliding surface and controller.

This paper is organized as follows. The description of time-delay systems and the problem formulation are given in next section. Section 3 is divided into three parts. In Subsection 3.1, the integral sliding surface is designed and the output feedback sliding mode control law is used to satisfy the reaching and sliding condition. Subsection 3.2 designs the full-order compensator and subsection 3.3 presents that the solutions to two algebraic Riccati inequalities guarantee the robust stability once the system is in the sliding mode. The feasibility of the proposed method is illustrated in Section 4 with numerical examples. Conclusions are given in Section 5.

\section{PROBLEM FORMULATION}

Consider a continuous-time time-delay system described by the state-space form as

$$
\begin{aligned}
\dot{\mathbf{x}}(t)= & (\mathbf{A}+\Delta \mathbf{A}(t)) \mathbf{x}(t)+\left(\mathbf{A}_{d}+\Delta \mathbf{A}_{d}(t)\right) \mathbf{x}(t-\tau) \\
& +\mathbf{B}(\mathbf{u}(t)+\mathbf{f}(\mathbf{x}, \mathbf{u}, t))+\mathbf{E} \mathbf{d}(t), \\
\mathbf{y}(t)= & \mathbf{C x}(t), \\
\mathbf{x}(t)= & \phi(t), \quad t \in[-\tau, 0],
\end{aligned}
$$

where $\mathbf{x} \in \mathbb{R}^{n}$ is the system state vector, $\mathbf{y} \in \mathbb{R}^{l}$ is the system output vector, $\mathbf{u} \in \mathbb{R}^{m}$ is the control input vector, and $\mathbf{d} \in \mathbb{R}^{p}$ is the mismatched disturbance vector. The function $\mathbf{f}(\mathbf{x}, \mathbf{u}, t) \in \mathbb{R}^{m}$ represents the unknown matched uncertainty. The constant $\tau$ is an unknown delay time but bounded by a known constant $\tau^{*}$, where $\tau \leq \tau^{*}$. The vector $\phi(t)$ is a continuous initial function. The real constant matrices $\mathbf{A}, \mathbf{A}_{d}, \boldsymbol{B}, \boldsymbol{E}$, and $\boldsymbol{C}$ are known and have appropriate dimensions with $l \geq m$. The structure uncertain- ties $\Delta \mathbf{A}(t)$ and $\Delta \mathbf{A}_{d}(t)$ satisfy $\Delta \mathbf{A}(t)=\mathbf{D} \Phi(t) \mathbf{H}$ and $\Delta \mathbf{A}_{d}(t)=\mathbf{D}_{d} \Phi_{d}(t) \mathbf{H}_{d}$, where $\mathbf{D}, \mathbf{D}_{d}, \mathbf{H}$, and $\mathbf{H}_{d}$ are non-unique known constant matrices with appropriate dimensions. Moreover, the matrices $\Phi(t)$ and $\Phi_{d}(t)$ are unknown, satisfying $\Phi^{T}(t) \Phi(t) \leq \mathbf{I}$ and $\Phi_{d}^{T}(t) \Phi_{d}(t)$ $\leq \mathbf{I}$ for all $t$, respectively. The controlled plant (1) can be rewritten as

$$
\begin{aligned}
\dot{\mathbf{x}}(t)= & (\mathbf{A}+\mathbf{D} \Phi(t) \mathbf{H}) \mathbf{x}(t)+\mathbf{B}(\mathbf{u}(t)+\mathbf{f}(\mathbf{x}, \mathbf{u}, t)) \\
& +\left(\mathbf{A}_{d}+\mathbf{D}_{d} \Phi_{d}(t) \mathbf{H}_{d}\right) \mathbf{x}(t-\tau)+\mathbf{E d}(t), \\
\mathbf{y}(t)= & \mathbf{C} \mathbf{x}(t) .
\end{aligned}
$$

Suppose that $(\mathbf{A}, \mathbf{B}, \mathbf{C})$ is completely controllable and observable. Edwards and Spurgeon [25] have shown that there exists a stable static output feedback sliding mode controller if

(C1) $\operatorname{rank}(\mathbf{C B})=\operatorname{rank}(\mathbf{B})=m$,

(C2) $(\mathbf{A}, \mathbf{B}, \mathbf{C})$ is minimum phase.

In the case of time-delay systems satisfying conditions (C1) and (C2), Castanos and Fridman [26] mentioned the state-dependent integral sliding surface design for linear systems with mismatched disturbances to ensure the robust disturbance attenuation. Niu et al. [16] proposed the observer-based sliding mode controller involving a synthesis condition to stabilize uncertain time-delay systems. Since the output is the only available signal, this paper presents the output-dependent integral sliding surface applying the full-order compensator in which the proposed control algorithm can guarantee the performance bound of robust disturbance attenuation $[22,23]$ once the system is in the sliding mode. The control algorithm involving the information of outputs and the compensator is designed to satisfy the reaching and sliding condition and to perform the controlled system entering the sliding mode without any synthesis condition. Before introducing main results, the following three assumptions are fulfilled throughout this paper.

Assumption 1: The matched term $\mathbf{f}(\mathbf{x}, \mathbf{u}, t)$ and mismatched disturbance $\mathbf{d}(t)$ are norm-bounded as

$$
\|\mathbf{f}(\mathbf{x}, \mathbf{u}, t)\| \leq \eta(t, \mathbf{y})+\chi\|\mathbf{u}(t)\| \text { and }\|\mathbf{d}(t)\| \leq \bar{d},
$$

where $0 \leq \chi<1, \eta(t, \mathbf{y})$, and $\bar{d}$ are known positive constants, respectively. The symbol $\|\bullet\|$ denotes the 2norm of $\bullet$.

Assumption 2: $(\mathbf{A}, \mathbf{B}, \mathbf{C})$ is minimum phase.

Assumption 3: $\operatorname{rank}(\mathbf{C B})=\operatorname{rank}(\mathbf{B})=m$.

\section{INTEGRAL SLIDING MODE CONTROLLER DESIGN}

In this section, the controller using the output only is first designed to force system (2) in the sliding mode from the initial moment. Then the output feedback integral sliding mode controller is proposed by employing the full-order compensator. Once the system is in the sliding mode, the proposed algorithm can guarantee the stability of the closed-loop system and 
sustain the nature of robust disturbance attenuation when two algebraic Riccati inequalities have solutions.

3.1. Integral sliding surface and sliding mode controller

Since Assumption 3 holds, we design the outputdependant integral sliding surface as

$$
\mathbf{s}(t)=(\mathbf{G C B})^{-1} \mathbf{G}(\mathbf{y}(t)-\mathbf{y}(0))-\int_{0}^{t} \mathbf{v}(q) d q,
$$

where $\mathbf{G} \in \mathbb{R}^{m \times l}$ is chosen such that $\mathbf{G C B}$ is invertible and $\mathbf{v} \in \mathbb{R}^{m}$ is designed later. Substituting system (2) into the derivative of $\mathbf{s}(t)$ with respect to time can obtain

$$
\begin{aligned}
\dot{\mathbf{s}}(t)= & \overline{\mathbf{G}}((\mathbf{A}+\mathbf{D} \Phi(t) \mathbf{H}) \mathbf{x}(t)+\mathbf{E d}(t) \\
& +\left(\mathbf{A}_{d}+\mathbf{D}_{d} \Phi_{d}(t) \mathbf{H}_{d}\right) \mathbf{x}(t-\tau) \\
& +\mathbf{B}(\mathbf{u}(t)+\mathbf{f}(\mathbf{x}, \mathbf{u}, t)))-\mathbf{v}(t) \\
= & \overline{\mathbf{G}}((\mathbf{A}+\mathbf{D} \Phi(t) \mathbf{H}) \mathbf{x}(t)+\mathbf{E d}(t) \\
& \left.+\left(\mathbf{A}_{d}+\mathbf{D}_{d} \Phi_{d}(t) \mathbf{H}_{d}\right) \mathbf{x}(t-\tau)\right)+\mathbf{u}(t) \\
& +\mathbf{f}(\mathbf{x}, \mathbf{u}, t)-\mathbf{v}(t),
\end{aligned}
$$

where $\overline{\mathbf{G}}=(\mathbf{G C B})^{-1} \mathbf{G C}$. Referring to [28], define two regions $\Omega_{1}$ and $\Omega_{2}$ as

$$
\begin{aligned}
\Omega_{1}= & \left\{\mathbf{x}(t) \mid\|\overline{\mathbf{G}}(\mathbf{A}+\mathbf{D} \Phi(t) \mathbf{H}) \mathbf{x}(t)\| \leq \sigma_{1}\right\} \subset \Omega, \\
\Omega_{2}= & \left\{\mathbf{x}(t-\tau) \mid\left\|\overline{\mathbf{G}}\left(\mathbf{A}_{d}+\mathbf{D}_{d} \Phi_{d}(t) \mathbf{H}_{d}\right) \mathbf{x}(t-\tau)\right\|\right. \\
& \left.\leq \sigma_{2}\right\} \subset \Omega,
\end{aligned}
$$

where $\sigma_{1}>0$ and $\sigma_{2}>0$ are known and bounded constants, and the region $\Omega \subset \mathbb{R}^{n}$ is a neighborhood of the origin. Consider system (2) in $\Omega_{1} \times \Omega_{2}$ and design the control input as

$$
\mathbf{u}(t)=\mathbf{v}(t)-\kappa(t) \mathbf{s}(t) /\|\mathbf{s}(t)\|,
$$

where $\kappa(t)=(1-\chi)^{-1}\left(\sigma_{1}+\sigma_{2}+\eta(t, \mathbf{y})+\chi\|\mathbf{v}(t)\|+\mu+\psi \bar{d}\right)$. The remaining parameters $\psi=\|\overline{\mathbf{G}} \mathbf{E}\|$ and $\mu$ are also positive constants. Through straightforward calculations, we know that

$$
\begin{aligned}
\kappa(t) & =\chi \kappa(t)+\chi\|\mathbf{v}(t)\|+\sigma_{1}+\sigma_{2}+\eta(t, \mathbf{y})+\psi \bar{d}+\mu \\
& \geq \chi\|\mathbf{u}(t)\|+\sigma_{1}+\sigma_{2}+\eta(t, \mathbf{y})+\psi \bar{d}+\mu .
\end{aligned}
$$

Substituting (6) into (4) can attain the following reaching and sliding condition:

$$
\begin{aligned}
\mathbf{s}^{T}(t) \dot{\mathbf{s}}(t)= & \mathbf{s}^{T}(t)(\overline{\mathbf{G}}(\mathbf{A}+\mathbf{D} \Phi(t) \mathbf{H}) \mathbf{x}(t)+\overline{\mathbf{G}} \mathbf{E d}(t) \\
& +\overline{\mathbf{G}}\left(\mathbf{A}_{d}+\mathbf{D}_{d} \Phi_{d}(t) \mathbf{H}_{d}\right) \mathbf{x}(t-\tau) \\
& +\mathbf{f}(\mathbf{x}, \mathbf{u}, t))-\kappa(t)\|\mathbf{s}(t)\| \\
\leq & \|\mathbf{S}(t)\|(\overline{\mathbf{G}}(\mathbf{A}+\mathbf{D} \Phi(t) \mathbf{H}) \mathbf{x}(t)+\overline{\mathbf{G}} \mathbf{E d}(t) \\
& +\overline{\mathbf{G}}\left(\mathbf{A}_{d}+\mathbf{D}_{d} \Phi_{d}(t) \mathbf{H}_{d}\right) \mathbf{x}(t-\tau) \\
& +\mathbf{f}(\mathbf{x}, \mathbf{u}, t)-\kappa(t))
\end{aligned}
$$

$$
\begin{aligned}
\leq & (\alpha\|\mathbf{x}(t)\|+\beta\|\mathbf{x}(t-\tau)\|+\eta(t, y)+\psi \bar{d} \\
& +\chi\|\mathbf{u}(t)\|-\kappa(t))\|\mathbf{s}(t)\| \\
\leq & \left(\alpha\|\mathbf{x}(t)\|+\beta\|\mathbf{x}(t-\tau)\|-\sigma_{1}-\sigma_{2}-\mu\right) \times\|\mathbf{s}(t)\| \\
\leq & -\mu\|\mathbf{s}(t)\|,
\end{aligned}
$$

where $\alpha=\|\overline{\mathbf{G}}(\mathbf{A}+\mathbf{D} \Phi(t) \mathbf{H})\|$ and $\beta=\| \overline{\mathbf{G}}\left(\mathbf{A}_{d}+\mathbf{D}_{d} \Phi_{d}(t)\right.$ $\left.\mathbf{H}_{d}\right) \|$. Since $\mathbf{s}(0)=\mathbf{0}$, the control input (6) can guarantee the following identities:

$$
\mathbf{s}(t)=\dot{\mathbf{s}}(t)=\mathbf{0} \forall \quad t \geq 0 .
$$

Therefore, the design of integral sliding surface (3) can shorten the transient time such that the system entered the sliding mode efficiently. Subsequently, this paper focuses on the stability analysis when the system is in the sliding mode.

From (4), once the system is in the sliding mode, $\mathbf{s}(t)=\dot{\mathbf{s}}(t)=\mathbf{0}$, the corresponding equivalent control [25] is given by

$$
\begin{aligned}
\mathbf{u}_{e q}(t)+\mathbf{f}\left(\mathbf{x}, \mathbf{u}_{e q}, t\right)= & -(\overline{\mathbf{G}}(\mathbf{A}+\mathbf{D} \Phi(t) \mathbf{H}) \mathbf{x}(t) \\
& +\overline{\mathbf{G}}\left(\left(\mathbf{A}_{d}+\mathbf{D}_{d} \Phi_{d}(t) \mathbf{H}_{d}\right) \mathbf{x}(t-\tau)\right. \\
& +\mathbf{E} \mathbf{d}(t)))+\mathbf{v}(t)
\end{aligned}
$$

Deriving the closed-loop system dynamics in the sliding mode from substituting (7) into system (2) can obtain

$$
\begin{aligned}
\dot{\mathbf{x}}(t)= & (\mathbf{A}+\mathbf{D} \Phi(t) \mathbf{H}) \mathbf{x}(t)+\mathbf{B} \mathbf{f}\left(\mathbf{x}, \mathbf{u}_{e q}, t\right) \\
& +\left(\mathbf{A}_{d}+\mathbf{D}_{d} \Phi_{d}(t) \mathbf{H}_{d}\right) \mathbf{x}(t-\tau)+\mathbf{B v}(t) \\
& -\mathbf{B} \mathbf{f}\left(\mathbf{x}, \mathbf{u}_{e q}, t\right)-\mathbf{B} \overline{\mathbf{G}}(\mathbf{A}+\mathbf{D} \Phi(t) \mathbf{H}) \mathbf{x}(t) \\
& -\mathbf{B} \overline{\mathbf{G}}\left(\mathbf{A}_{d}+\mathbf{D}_{d} \Phi_{d}(t) \mathbf{H}_{d}\right) \mathbf{x}(t-\tau) \\
& -\mathbf{B} \overline{\mathbf{G}} \mathbf{E} \mathbf{d}(t)+\mathbf{E d}(t) \\
= & \left(\mathbf{I}_{n}-\mathbf{B} \overline{\mathbf{G}}\right)((\mathbf{A}+\mathbf{D} \Phi(t) \mathbf{H}) \mathbf{x}(t)+\mathbf{E d}(t) \\
& \left.+\left(\mathbf{A}_{d}+\mathbf{D}_{d} \Phi_{d}(t) \mathbf{H}_{d}\right) \mathbf{x}(t-\tau)\right)+\mathbf{B v}(t) \\
= & \mathbf{N}(\mathbf{A}+\mathbf{D} \Phi(t) \mathbf{H}) \mathbf{x}(t)+\mathbf{N E d}(t)+\mathbf{B v}(t) \\
& +\mathbf{N}\left(\mathbf{A}_{d}+\mathbf{D}_{d} \Phi_{d}(t) \mathbf{H}_{d}\right) \mathbf{x}(t-\tau)
\end{aligned}
$$

where $\mathbf{N}=\mathbf{I}_{n}-\mathbf{B} \overline{\mathbf{G}}$. Since $\mathbf{N A}=\mathbf{A}-\mathbf{B} \overline{\mathbf{G}} \mathbf{A}$, we have the following relationship:

$$
\begin{aligned}
{\left[\begin{array}{ll}
s \mathbf{I}_{n}-\mathbf{N A} & \mathbf{B}
\end{array}\right] } & =\left[\begin{array}{ll}
s \mathbf{I}_{n}-\mathbf{A}+\mathbf{B} \overline{\mathbf{G}} \mathbf{A} & \mathbf{B}
\end{array}\right] \\
& =\left[\begin{array}{ll}
s \mathbf{I}_{n}-\mathbf{A} & \mathbf{B}
\end{array}\right]\left[\begin{array}{cc}
\mathbf{I}_{n} & \mathbf{0} \\
\overline{\mathbf{G}}^{\mathbf{A}} & \mathbf{I}_{n}
\end{array}\right] .
\end{aligned}
$$

Since the pair $(\mathbf{A}, \mathbf{B})$ is controllable, $\operatorname{rank}\left(\left[s \mathbf{I}_{n}-\mathbf{A} \mathbf{B}\right]\right)$ $=n$ for $s \in \mathbb{C}$, the controllability of $(\mathbf{N A}, \mathbf{B})$ can be guaranteed by

$$
\begin{aligned}
& \operatorname{rank}\left(\left[s \mathbf{I}_{n}-\mathbf{N A} \quad \mathbf{B}\right]\right) \\
& \quad=\operatorname{rank}\left(\left[\begin{array}{ll}
s \mathbf{I}_{n}-\mathbf{A} & \mathbf{B}
\end{array}\right]\left[\begin{array}{cc}
\mathbf{I}_{n} & \mathbf{0} \\
\overline{\mathbf{G}} \mathbf{A} & \mathbf{I}_{n}
\end{array}\right]\right)=n
\end{aligned}
$$


for $s \in \mathbb{C}$. It implies that the pair (NA, B) is also controllable. Referring to $[22,23]$, the next step for the robust disturbance attenuation correlated with system (8) is to design $\mathbf{v}(t)$ such that the system is stable and satisfies the following inequality:

$$
\int_{0}^{t}\left(\mathbf{y}^{T} \mathbf{y}+\mathbf{v}^{T} \mathbf{R v}\right) d q \leq \gamma^{2} \int_{0}^{t}\left(\mathbf{d}^{T} \mathbf{d}\right) d q \forall t \geq 0,
$$

where $0 \leq \gamma<\infty$ and $\mathbf{R}>0$ is a weighting matrix. Next subsection will utilize a full-order compensator to complete the design of $\mathbf{v}(t)$ fulfilling the robust disturbance attenuation.

Remark 1: In [26], the integration term in the sliding manifold can be thought as a trajectory of the system in the absence of perturbations and in the presence of the nominal control, i.e., as a nominal trajectory for a given initial condition. In this paper, adding the integration term $\mathbf{v}(t)$ into the sliding surface (3) can compensate the degree of freedom to attenuate the effects of disturbances and uncertainties in the closed-loop system. Involving the integrator is also helpful to analyze the stability and robustness of the closed-loop system.

Remark 2: The assumption of upper bounds for sufficient conditions (5) is a conventional issue for output feedback sliding mode controller designs. It causes the local reaching and sliding condition. For reducing the corresponding conservatism, an adaptation law or a filter can estimate a bound of norm of some unknown states to release conditions (5) and satisfy the global reaching and sliding condition, if the delay time $\tau$ is known.

\subsection{Full-order compensator}

Before designing the compensator, define matrices as

$$
\begin{aligned}
& \mathbf{U}=-\mathbf{B}(\mathbf{C B})^{+}+\mathbf{Y}\left(\mathbf{I}_{l}-\mathbf{C B}(\mathbf{C B})^{+}\right), \\
& \mathbf{M}=\mathbf{I}_{n}+\mathbf{U C}, \\
& \Gamma=\mathbf{L}\left(\mathbf{I}_{l}+\mathbf{C U}\right)-\mathbf{M A U},
\end{aligned}
$$

where $(\mathbf{C B})^{+}=\left((\mathbf{C B})^{T} \mathbf{C B}\right)^{-1}(\mathbf{C B})^{T}, \quad \mathbf{Y} \in \mathbb{R}^{n \times l}$ is an arbitrary matrix, and $\boldsymbol{L}$ is a gain matrix designed later. Notice that the product of MB is given by

$$
\begin{aligned}
\mathbf{M B} & =\mathbf{B}+\mathbf{U C B} \\
& =\mathbf{B}-\mathbf{B}(\mathbf{C B})^{+} \mathbf{C B}+\mathbf{Y}\left(\mathbf{C B}-\mathbf{C B}(\mathbf{C B})^{+} \mathbf{C B}\right)=\mathbf{0}
\end{aligned}
$$

and $\operatorname{rank}(\mathbf{M})=n-m$ from Assumption 3. Then the function $\mathbf{v}(t)$ is generated from the following full-order dynamic compensator:

$$
\begin{aligned}
& \dot{\xi}(t)=(\mathbf{M A}-\mathbf{L C}+\mathbf{F}) \xi(t)+(\Gamma-\mathbf{F U}) \mathbf{y}(t), \\
& \mathbf{v}(t)=-\mathbf{K}(\xi(t)-\mathbf{U y}(t))
\end{aligned}
$$

where $\xi \in \mathbb{R}^{n}$ is an available vector of auxiliary states. Moreover, $\mathbf{K} \in \mathbb{R}^{m \times n}$ and $\mathbf{F} \in \mathbb{R}^{n \times n}$ are gain matrices decided later. According to (10) and $\mathbf{M B}=\mathbf{0}$, the dynamics of error vector $\mathbf{e}=\mathbf{M x}-\xi$ can be given by

$$
\begin{aligned}
\dot{\mathbf{e}}(t)= & \mathbf{M}((\mathbf{A}+\mathbf{D} \Phi(t) \mathbf{H}) \mathbf{x}(t)+\mathbf{E d}(t)) \\
& +\left(\mathbf{A}_{d}+\mathbf{D}_{d} \Phi_{d}(t) \mathbf{H}_{d}\right) \mathbf{x}(t-\tau) \\
& -(\mathbf{M A}-\mathbf{L C}+\mathbf{F}) \xi(t)-(\Gamma-\mathbf{F U}) \mathbf{y}(t) \\
= & (\mathbf{M A}-\mathbf{L C}) \mathbf{M x}(t)-(\mathbf{M A}-\mathbf{L C}) \xi(t) \\
& +\mathbf{M}\left(\mathbf{A}_{d}+\mathbf{D}_{d} \Phi_{d}(t) \mathbf{H}_{d}\right) \mathbf{x}(t-\tau)+\mathbf{F e}(t) \\
& -\mathbf{F x}(t)+\mathbf{M D} \Phi(t) \mathbf{H} \mathbf{x}(t)+\mathbf{M E d}(t) \\
= & (\mathbf{M D} \Phi(t) \mathbf{H}-\mathbf{F}) \mathbf{x}(t) \\
& +\mathbf{M}\left(\mathbf{A}_{d}+\mathbf{D}_{d} \Phi_{d}(t) \mathbf{H}_{d}\right) \mathbf{x}(t-\tau) \\
& +(\mathbf{M A}-\mathbf{L C}+\mathbf{F}) \mathbf{e}(t)+\mathbf{M E d}(t) .
\end{aligned}
$$

On the other hand, $\mathbf{v}(t)$ can be rewritten as

$$
\mathbf{v}(t)=-\mathbf{K x}(t)+\mathbf{K e}(t) .
$$

Substituting (12) into (8) can obtain the system dynamics in the sliding mode as

$$
\begin{aligned}
\dot{\mathbf{x}}(t)= & (\mathbf{N}(\mathbf{A}+\mathbf{D} \Phi(t) \mathbf{H})-\mathbf{B K}) \mathbf{x}(t)+\mathbf{B K e}(t) \\
& +\mathbf{N}\left(\mathbf{A}_{d}+\mathbf{D}_{d} \Phi_{d}(t) \mathbf{H}_{d}\right) \mathbf{x}(t-\tau)+\mathbf{N E d}(t) .
\end{aligned}
$$

Combining (11) with (13), the overall closed-loop system is shown as below:

$$
\begin{aligned}
& {\left[\begin{array}{c}
\dot{\mathbf{x}}(t) \\
\dot{\mathbf{e}}(t)
\end{array}\right]=\left[\begin{array}{c}
\mathbf{N A}-\mathbf{B K}+\mathbf{N D} \Phi(t) \mathbf{H} \\
-\mathbf{F}+\mathbf{M D} \Phi(t) \mathbf{H}
\end{array}\right.} \\
& \left.\begin{array}{c}
\mathbf{B K} \\
\mathbf{M A}-\mathbf{L C}+\mathbf{F}
\end{array}\right]\left[\begin{array}{l}
\mathbf{x}(t) \\
\mathbf{e}(t)
\end{array}\right]+\left[\begin{array}{l}
\mathbf{N E} \\
\mathbf{M E}
\end{array}\right] \mathbf{d}(t) \\
& +\left[\begin{array}{cc}
\mathbf{N} \mathbf{A}_{d}+\mathbf{N D}_{d} \Phi_{d}(t) \mathbf{H}_{d} & \mathbf{0} \\
\mathbf{M A}_{d}+\mathbf{M D}_{d} \Phi_{d}(t) \mathbf{H}_{d} & \mathbf{0}
\end{array}\right]\left[\begin{array}{l}
\mathbf{x}(t-\tau) \\
\mathbf{e}(t-\tau)
\end{array}\right] .
\end{aligned}
$$

Moreover, to represent the term $\mathbf{y}^{T} \mathbf{y}+\mathbf{v}^{T} \mathbf{R v}$ in (9), we define the controlled output $\mathbf{z} \in \mathbb{R}^{l+m}$ as

$$
\begin{aligned}
\mathbf{z}(t) & =\left[\begin{array}{ll}
\mathbf{C}^{T} & \mathbf{0}
\end{array}\right] \mathbf{x}(t)+\left[\begin{array}{ll}
\mathbf{0} & \mathbf{C}_{v}^{T}
\end{array}\right] \mathbf{v}(t) \\
& =\left[\begin{array}{ll}
\mathbf{C}^{T} & -\mathbf{K}^{T} \mathbf{C}_{v}^{T}
\end{array}\right] \mathbf{x}(t)+\left[\begin{array}{ll}
\mathbf{0} & \mathbf{K}^{T} \mathbf{C}_{v}^{T}
\end{array}\right] \mathbf{e}(t) \\
& =\overline{\mathbf{C}} \mathbf{x}(t)+\overline{\mathbf{C}}_{v} \mathbf{e}(t)=\mathbf{C}_{w}\left[\begin{array}{ll}
\mathbf{x}^{T}(t) & \mathbf{e}^{T}(t)
\end{array}\right]
\end{aligned}
$$

where $\quad \mathbf{C}_{v}^{T} \mathbf{C}_{v}=\mathbf{R}$ and $\mathbf{C}_{w}=\left[\begin{array}{l}\overline{\mathbf{C}}^{T} \\ \overline{\mathbf{C}}_{v}^{T}\end{array}\right]=\left[\begin{array}{cc}\mathbf{C} & \mathbf{0} \\ -\mathbf{C}_{v} \mathbf{K} & \mathbf{C}_{v} \mathbf{K}\end{array}\right]$.

The auxiliary matrix $\mathbf{C}_{v}$ is implicit and does not appear in the controller, but $\mathbf{C}_{v}^{T} \mathbf{C}_{v}$ is defined as the matrix $\boldsymbol{R}$ which is the weighting gain of $\mathbf{v}(t)$. Next subsection will design matrices $\boldsymbol{K}, \boldsymbol{L}$, and $\boldsymbol{F}$, and analyze the robust stability of the closed-loop system (14).

\subsection{Robust disturbance attenuation}

Define a quadratic energy function as

$$
\begin{aligned}
E_{n}(\mathbf{x}, \mathbf{e})= & \mathbf{x}^{T}(t) \mathbf{P}_{1} \mathbf{x}(t)+\mathbf{e}^{T}(t) \mathbf{P}_{2} \mathbf{e}(t) \\
& +\int_{t-\tau}^{t} \mathbf{x}^{T}(\alpha) \mathbf{Q}_{1} \mathbf{x}(\alpha) d \alpha \\
& +\int_{t-\tau}^{t} \mathbf{e}^{T}(\alpha) \mathbf{Q}_{2} \mathbf{e}(\alpha) d \alpha,
\end{aligned}
$$


where the matrices $\mathbf{P}_{1}>0, \mathbf{P}_{2}>0, \mathbf{Q}_{1}>0$, and $\mathbf{Q}_{2}>0$ are determined later. Then define the Hamiltonian function as

$$
H[\mathbf{d}]=\mathbf{z}^{T} \mathbf{z}-\gamma^{2} \mathbf{d}^{T} \mathbf{d}+d E_{n} / d t,
$$

where $d E_{n} / d t$ is the derivative of $E_{n}$ along the trajectory of the closed-loop system (14). A sufficient condition satisfying the robust disturbance attenuation is that

$$
H[\mathbf{d}]<0 \text {, for all } \mathbf{d} \in L_{2}[0, \infty),
$$

where $L_{2}$ is a Hilbert space of matrix-valued (or scalarvalued) function and consists of all functions $\mathbf{d}(s)$ such that $\int_{0}^{\infty} \operatorname{trace}\left[\mathbf{d}^{T}(s) \mathbf{d}(s)\right] d s<\infty$ is bounded. Since (18) holds, $E_{n}(\mathbf{x}, \mathbf{e})$ is a strict radially unbounded Lyapunov function of the closed-loop system (14), and hence the robust stability can be guaranteed [22]. Notice that (18) is equivalent to $\sup H[\mathbf{d}]<0$. As $\mathbf{z}(t)=\overline{\mathbf{C}} \mathbf{x}(t)+\overline{\mathbf{C}}_{v} \mathbf{e}(t)$, $\mathbf{d} \in L_{2}$

(17) can be rewritten as

$$
\begin{aligned}
H[\mathbf{d}]= & \mathbf{x}^{T}(t)\left(\mathbf{C}^{T} \mathbf{C}+\mathbf{K}^{T} \mathbf{R K}\right) \mathbf{x}(t)-\gamma^{2} \mathbf{d}^{T}(t) \mathbf{d}(t) \\
& -2 \mathbf{x}^{T}(t) \mathbf{K}^{T} \mathbf{R K e}(t)+\mathbf{e}^{T}(t) \mathbf{K}^{T} \mathbf{R K e}(t) \\
& +2((\mathbf{N A}-\mathbf{B K}+\mathbf{N D} \Phi(t) \mathbf{H}) \mathbf{x}(t))^{T} \mathbf{P}_{1} \mathbf{x}(t) \\
& +2(\mathbf{B K e}(t))^{T} \mathbf{P}_{1} \mathbf{x}(t)+2 \mathbf{e}^{T}(t) \mathbf{P}_{2} \mathbf{M E d}(t) \\
& +2\left(\mathbf{N}\left(\mathbf{A}_{d}+\mathbf{D}_{d} \Phi_{d}(t) \mathbf{H}_{d}\right) \mathbf{x}(t-\tau)\right)^{T} \mathbf{P}_{1} \mathbf{x}(t) \\
& +2((\mathbf{M D} \Phi(t) \mathbf{H}-\mathbf{F}) \mathbf{x}(t))^{T} \mathbf{P}_{2} \mathbf{e}(t) \\
& +2((\mathbf{M A}-\mathbf{L C}+\mathbf{F}) \mathbf{e}(t))^{T} \mathbf{P}_{2} \mathbf{e}(t) \\
& +2\left(\mathbf{M}\left(\mathbf{A}_{d}+\mathbf{D}_{d} \Phi_{d}(t) \mathbf{H}_{d}\right) \mathbf{x}(t-\tau)\right)^{T} \mathbf{P}_{2} \mathbf{e}(t) \\
& +\mathbf{x}^{T}(t) \mathbf{Q}_{1} \mathbf{x}(t)-\mathbf{x}^{T}(t-\tau) \mathbf{Q}_{1} \mathbf{x}(t-\tau) \\
& +\mathbf{e}^{T}(t) \mathbf{Q}_{2} \mathbf{e}(t)-\mathbf{e}^{T}(t-\tau) \mathbf{Q}_{2} \mathbf{e}(t-\tau) \\
& +2 \mathbf{x}^{T}(t) \mathbf{P}_{1} \mathbf{N E d}(t) .
\end{aligned}
$$

Based on the above equation, the worst case of sup $H[\mathbf{d}]$ occurs when $\mathbf{d} \in L_{2}$

$$
\mathbf{d}(t)=\gamma^{-2} \mathbf{E}^{T}\left(\mathbf{N}^{T} \mathbf{P}_{1} \mathbf{x}(t)+\mathbf{M}^{T} \mathbf{P}_{2} \mathbf{e}(t)\right)
$$

and it follows that

$$
\begin{aligned}
H[\mathbf{d}] \leq & \mathbf{x}^{T}(t)\left(\mathbf{C}^{T} \mathbf{C}+\mathbf{K}^{T} \mathbf{R K}\right) \mathbf{x}(t) \\
& -2 \mathbf{e}^{T}(t) \mathbf{K}^{T} \mathbf{R K} \mathbf{x}(t)+\mathbf{e}^{T}(t) \mathbf{K}^{T} \mathbf{R K e}(t) \\
& +2((\mathbf{N A}-\mathbf{B K}+\mathbf{N D} \Phi(t) \mathbf{H}) \mathbf{x}(t))^{T} \mathbf{P}_{1} \mathbf{x}(t) \\
& +2(\mathbf{B K e}(t))^{T} \mathbf{P}_{1} \mathbf{x}(t) \\
& +2\left(\mathbf{N}\left(\mathbf{A}_{d}+\mathbf{D}_{d} \Phi_{d}(t) \mathbf{H}_{d}\right) \mathbf{x}(t-\tau)\right)^{T} \mathbf{P}_{1} \mathbf{x}(t) \\
& +2((\mathbf{M D} \Phi(t) \mathbf{H}-\mathbf{F}) \mathbf{x}(t))^{T} \mathbf{P}_{2} \mathbf{e}(t) \\
& +2((\mathbf{M A}-\mathbf{L C}+\mathbf{F}) \mathbf{e}(t))^{T} \mathbf{P}_{2} \mathbf{e}(t) \\
& +2\left(\mathbf{M}\left(\mathbf{A}_{d}+\mathbf{D}_{d} \Phi_{d}(t) \mathbf{H}_{d}\right) \mathbf{x}(t-\tau)\right)^{T} \mathbf{P}_{2} \mathbf{e}(t) \\
& +\mathbf{x}^{T}(t) \mathbf{Q}_{1} \mathbf{x}(t)-\mathbf{x}^{T}(t-\tau) \mathbf{Q}_{1} \mathbf{x}(t-\tau)
\end{aligned}
$$

$$
\begin{aligned}
& +\mathbf{e}^{T}(t) \mathbf{Q}_{2} \mathbf{e}(t)-\mathbf{e}^{T}(t-\tau) \mathbf{Q}_{2} \mathbf{e}(t-\tau) \\
& +\gamma^{-2} \mathbf{x}^{T}(t) \mathbf{P}_{1} \mathbf{N E E}^{T} \mathbf{N}^{T} \mathbf{P}_{1} \mathbf{x}(t) \\
& +2 \gamma^{-2} \mathbf{e}^{T}(t) \mathbf{P}_{2} \mathbf{M E E}^{T} \mathbf{N}^{T} \mathbf{P}_{1} \mathbf{x}(t) \\
& +\gamma^{-2} \mathbf{e}^{T}(t) \mathbf{P}_{2} \mathbf{M E E}^{T} \mathbf{M}^{T} \mathbf{P}_{2} \mathbf{e}(t) .
\end{aligned}
$$

The following lemma is introduced to obtain the bound of the uncertainty variations in (19) by known quantities.

Lemma [17]: Given real matrices $\boldsymbol{D}, \Phi(t)$, and $\boldsymbol{H}$ of appropriate dimensions, suppose $\Phi^{T}(t) \Phi(t) \leq \mathbf{I}$, for any positive scalar $\rho$, then

$$
\mathbf{D} \Phi(t) \mathbf{H}+\mathbf{H}^{T} \Phi^{T}(t) \mathbf{D}^{T} \leq \rho \mathbf{D} \mathbf{D}^{T}+\rho^{-1} \mathbf{H}^{T} \mathbf{H} .
$$

According to the previous lemma, inequality (19) can be rewritten as

$$
\begin{aligned}
& H[\mathbf{d}] \leq \mathbf{x}^{T}(t)\left((\mathbf{N A}-\mathbf{B K})^{T} \mathbf{P}_{1}+\mathbf{P}_{1}(\mathbf{N A}-\mathbf{B K})\right) \mathbf{x}(t) \\
& +\mathbf{x}^{T}(t)\left(\mathbf{C}^{T} \mathbf{C}+\mathbf{K}^{T} \mathbf{R K}+\mathbf{Q}_{1}\right) \mathbf{x}(t) \\
& +\mathbf{x}^{T}(t) \mathbf{P}_{1} \mathbf{N}\left(\gamma^{-2} \mathbf{E} \mathbf{E}^{T}+\rho_{1} \mathbf{D D}^{T}\right) \mathbf{N}^{T} \mathbf{P}_{1} \mathbf{x}(t) \\
& +\rho_{2} \mathbf{x}^{T}(t) \mathbf{P}_{1} \mathbf{N D}_{d} \mathbf{D}_{d}^{T} \mathbf{N}^{T} \mathbf{P}_{1} \mathbf{x}(t) \\
& +\mathbf{x}^{T}(t)\left(\left(\rho_{1}^{-1}+\rho_{3}^{-1}\right) \mathbf{H}^{T} \mathbf{H}\right) \mathbf{x}(t) \\
& +\mathbf{e}^{T}(t)(\mathbf{M A}-\mathbf{L C}+\mathbf{F})^{T} \mathbf{P}_{2} \mathbf{e}(t) \\
& +\mathbf{e}^{T}(t) \mathbf{P}_{2}(\mathbf{M A}-\mathbf{L C}+\mathbf{F}) \mathbf{e}(t) \\
& +\mathbf{e}^{T}(t)\left(\mathbf{K}^{T} \mathbf{R K}+\mathbf{Q}_{2}\right) \mathbf{e}(t) \\
& +\mathbf{e}^{T}(t) \mathbf{P}_{2} \mathbf{M}\left(\gamma^{-2} \mathbf{E E}^{T}+\rho_{3} \mathbf{D} \mathbf{D}^{T}\right) \mathbf{M}^{T} \mathbf{P}_{2} \mathbf{e}(t) \\
& +\rho_{4} \mathbf{e}^{T}(t) \mathbf{P}_{2} \mathbf{M D}_{d} \mathbf{D}_{d}^{T} \mathbf{M}^{T} \mathbf{P}_{2} \mathbf{e}(t) \\
& -2 \mathbf{e}^{T}(t) \mathbf{K}^{T} \mathbf{R K x}(t)+2 \mathbf{e}^{T}(t)(\mathbf{B K})^{T} \mathbf{P}_{1} \mathbf{x}(t) \\
& -2 \mathbf{e}^{T}(t) \mathbf{P}_{2} \mathbf{F} \mathbf{x}(t)-\mathbf{e}^{T}(t-\tau) \mathbf{Q}_{2} \mathbf{e}(t-\tau) \\
& +2 \mathbf{e}^{T}(t) \gamma^{-2} \mathbf{P}_{2} \mathbf{M E E}^{T} \mathbf{N}^{T} \mathbf{P}_{1} \mathbf{x}(t) \\
& +2 \mathbf{x}^{T}(t-\tau)\left(\mathbf{N} \mathbf{A}_{d}\right)^{T} \mathbf{P}_{1} \mathbf{x}(t) \\
& +2 \mathbf{x}^{T}(t-\tau)\left(\mathbf{M A}_{d}\right)^{T} \mathbf{P}_{2} \mathbf{e}(t) \\
& +\mathbf{x}^{T}(t-\tau)\left(\left(\rho_{2}^{-1}+\rho_{4}^{-1}\right) \mathbf{H}_{d}^{T} \mathbf{H}_{d}-\mathbf{Q}_{1}\right) \mathbf{x}(t-\tau) \\
& =\left[\begin{array}{c}
\mathbf{x}(t) \\
\mathbf{e}(t) \\
\mathbf{x}(t-\tau) \\
\mathbf{e}(t-\tau)
\end{array}\right]^{T}\left[\begin{array}{cc}
\Pi_{11} & \Pi_{12} \\
\Pi_{12}^{T} & \Pi_{22} \\
\mathbf{A}_{d}^{T} \mathbf{N}^{T} \mathbf{P}_{1} & \mathbf{A}_{d}^{T} \mathbf{M}^{T} \mathbf{P}_{1} \\
\mathbf{0} & \mathbf{0}
\end{array}\right. \\
& \left.\begin{array}{cc}
\mathbf{P}_{1} \mathbf{N} \mathbf{A}_{d} & \mathbf{0} \\
\mathbf{P}_{2} \mathbf{M} \mathbf{A}_{d} & \mathbf{0} \\
\left(\rho_{2}^{-1}+\rho_{4}^{-1}\right) \mathbf{H}_{d}^{T} \mathbf{H}_{d}-\mathbf{Q}_{1} & \mathbf{0} \\
\mathbf{0} & -\mathbf{Q}_{2}
\end{array}\right]\left[\begin{array}{c}
\mathbf{x}(t) \\
\mathbf{e}(t) \\
\mathbf{x}(t-\tau) \\
\mathbf{e}(t-\tau)
\end{array}\right],
\end{aligned}
$$

where

$$
\begin{aligned}
\Pi_{11}= & (\mathbf{N A}-\mathbf{B K})^{T} \mathbf{P}_{1}+\mathbf{P}_{1}(\mathbf{N A}-\mathbf{B K}) \\
& +\mathbf{P}_{1} \mathbf{N}\left(\gamma^{-2} \mathbf{E} \mathbf{E}^{T}+\rho_{1} \mathbf{D} \mathbf{D}^{T}+\rho_{2} \mathbf{D}_{d} \mathbf{D}_{d}^{T}\right) \mathbf{N}^{T} \mathbf{P}_{1} \\
& +\mathbf{C}^{T} \mathbf{C}+\mathbf{K}^{T} \mathbf{R K}+\mathbf{Q}_{1}+\left(\rho_{1}^{-1}+\rho_{3}^{-1}\right) \mathbf{H}^{T} \mathbf{H}
\end{aligned}
$$




$$
\begin{aligned}
\Pi_{22}= & (\mathbf{M A}-\mathbf{L C}+\mathbf{F})^{T} \mathbf{P}_{2}+\mathbf{P}_{2}(\mathbf{M A}-\mathbf{L} \mathbf{C}+\mathbf{F}) \\
& +\mathbf{P}_{2} \mathbf{M}\left(\gamma^{-2} \mathbf{E} \mathbf{E}^{T}+\rho_{3} \mathbf{D D} \mathbf{D}^{T}+\rho_{4} \mathbf{D}_{d} \mathbf{D}_{d}^{T}\right) \mathbf{M}^{T} \mathbf{P}_{2} \\
& +\mathbf{K}^{T} \mathbf{R K}+\mathbf{Q}_{2}, \\
\Pi_{12}= & \mathbf{P}_{1} \mathbf{B K}-\mathbf{K}^{T} \mathbf{R K}-\mathbf{F}^{T} \mathbf{P}_{2}+\gamma^{-2} \mathbf{P}_{1} \mathbf{N} \mathbf{E} \mathbf{E}^{T} \mathbf{M}^{T} \mathbf{P}_{2} .
\end{aligned}
$$

Notice that $\rho_{i}$ are positive constants, $i=1, \cdots, 4$. The sufficient condition satisfying the robust disturbance attenuation, $\sup H[\mathbf{d}]<0$, is altered to fulfill the fol$\mathbf{d} \in L_{2}$

lowing matrix inequality:

$$
\left[\begin{array}{cccc}
\Pi_{11} & \Pi_{12} & \mathbf{P}_{1} \mathbf{N} \mathbf{A}_{d} & \mathbf{0} \\
\Pi_{12}^{T} & \Pi_{22} & \mathbf{P}_{2} \mathbf{M} \mathbf{A}_{d} & \mathbf{0} \\
\mathbf{A}_{d}^{T} \mathbf{N}^{T} \mathbf{P}_{1} & \mathbf{A}_{d}^{T} \mathbf{M}^{T} \mathbf{P}_{2} & -\left(\mathbf{Q}_{1}-\left(\rho_{2}^{-1}+\rho_{4}^{-1}\right) \mathbf{H}_{d}^{T} \mathbf{H}_{d}\right) & \mathbf{0} \\
\mathbf{0} & \mathbf{0} & \mathbf{0} & -\mathbf{Q}_{2}
\end{array}\right]
$$

Moreover, the following theorem transfers (20) into two algebraic Riccati inequalities using Schur decomposition and demonstrates the designs of $\boldsymbol{K}, \boldsymbol{L}$, and $\boldsymbol{F}$ which guarantee the robust disturbance attenuation.

Theorem 1: Consider system (8) with the full-order compensator (10). Given $\mathbf{Q}_{1}>0, \mathbf{Q}_{2}>0$, and $\rho_{i}>0$, $i=1,2, \cdots, 4$, if there exists matrices $\mathbf{P}_{1}>0$ and $\mathbf{P}_{2}>0$ satisfying the following algebraic Riccati inequalities

$$
\begin{aligned}
& (\mathbf{N A})^{T} \mathbf{P}_{1}+\mathbf{P}_{1}(\mathbf{N A})-\mathbf{P}_{1} \mathbf{B} \mathbf{R}^{-1} \mathbf{B}^{T} \mathbf{P}_{1}+\mathbf{C}^{T} \mathbf{C}+\mathbf{Q}_{1} \\
& \quad+\left(\rho_{1}^{-1}+\rho_{3}^{-1}\right) \mathbf{H}^{T} \mathbf{H}+\gamma^{-2} \mathbf{P}_{1} \mathbf{N E} \mathbf{E}^{T} \mathbf{N}^{T} \mathbf{P}_{1} \\
& \quad+\mathbf{P}_{1} \mathbf{N}\left(\rho_{1} \mathbf{D} \mathbf{D}^{T}+\rho_{2} \mathbf{D}_{d} \mathbf{D}_{d}^{T}\right) \mathbf{N}^{T} \mathbf{P}_{1}-\mathbf{P}_{1} \mathbf{N} \mathbf{A}_{d} \\
& \quad \times\left(\left(\rho_{2}^{-1}+\rho_{4}^{-1}\right) \mathbf{H}_{d}^{T} \mathbf{H}_{d}-\mathbf{Q}_{1}\right)^{-1} \mathbf{A}_{d}^{T} \mathbf{N}^{T} \mathbf{P}_{1}<0, \\
& (\mathbf{M A}+\mathbf{F})^{T} \mathbf{P}_{2}+\mathbf{P}_{2}(\mathbf{M A}+\mathbf{F})+\mathbf{P}_{1} \mathbf{B} \mathbf{R}^{-1} \mathbf{B}^{T} \mathbf{P}_{1} \\
& \quad-\lambda \mathbf{C}^{T} \mathbf{C}+\mathbf{Q}_{2}+\mathbf{P}_{2} \mathbf{M}\left(\gamma^{-2} \mathbf{E} \mathbf{E}^{T}+\rho_{3} \mathbf{D} \mathbf{D}^{T}\right) \mathbf{M}^{T} \mathbf{P}_{2} \\
& \quad+\rho_{4} \mathbf{P}_{2} \mathbf{M} \mathbf{D}_{d} \mathbf{D}_{d}^{T} \mathbf{M}^{T} \mathbf{P}_{2}-\mathbf{P}_{2} \mathbf{M} \mathbf{A}_{d} \\
& \quad \times\left(\left(\rho_{2}^{-1}+\rho_{4}^{-1}\right) \mathbf{H}_{d}^{T} \mathbf{H}_{d}-\mathbf{Q}_{1}\right)^{-1} \mathbf{A}_{d}^{T} \mathbf{M}^{T} \mathbf{P}_{2}<0,
\end{aligned}
$$

where $\rho_{2}>0$ and $\rho_{4}>0$ are designed such that $\mathbf{Q}_{1}-$ $\left(\rho_{2}^{-1}+\rho_{4}^{-1}\right) \mathbf{H}_{d}^{T} \mathbf{H}_{d}>0$, then robust disturbance attenuation (9) can be guaranteed. Furthermore, matrices $\boldsymbol{K}, \boldsymbol{L}$, and $\boldsymbol{F}$ are given by

$$
\begin{aligned}
\mathbf{K}= & \mathbf{R}^{-1} \mathbf{B}^{T} \mathbf{P}_{1}, \\
\mathbf{L}= & \lambda \mathbf{P}_{2}^{-1} \mathbf{C}^{T} / 2, \\
\mathbf{F}= & -\mathbf{M} \mathbf{A}_{d}\left(\left(\rho_{2}^{-1}+\rho_{4}^{-1}\right) \mathbf{H}_{d}^{T} \mathbf{H}_{d}-\mathbf{Q}_{1}\right)^{-1} \mathbf{A}_{d}^{T} \mathbf{N}^{T} \mathbf{P}_{1} \\
& +\gamma^{-2} \mathbf{M E} \mathbf{E}^{T} \mathbf{N}^{T} \mathbf{P}_{1} .
\end{aligned}
$$

Proof: Since the condition $\left(\rho_{2}^{-1}+\rho_{4}^{-1}\right) \mathbf{H}_{d}^{T} \mathbf{H}_{d}<\mathbf{Q}_{1}$ holds, by Schur decomposition, inequality (20) is equivalent to

$$
\left[\begin{array}{ll}
\mathbf{J}_{11} & \mathbf{J}_{12} \\
\mathbf{J}_{12}^{T} & \mathbf{J}_{22}
\end{array}\right]<0,
$$

where

$$
\begin{aligned}
\mathbf{J}_{11}= & (\mathbf{N A}-\mathbf{B K})^{T} \mathbf{P}_{1}+\mathbf{P}_{1}(\mathbf{N A}-\mathbf{B K})+\mathbf{K}^{T} \mathbf{R} \mathbf{K} \\
& +\mathbf{P}_{1} \mathbf{N}\left(\gamma^{-2} \mathbf{E} \mathbf{E}^{T}+\rho_{1} \mathbf{D} \mathbf{D}^{T}+\rho_{2} \mathbf{D}_{d} \mathbf{D}_{d}^{T}\right) \mathbf{N}^{T} \mathbf{P}_{1} \\
& +\mathbf{C}^{T} \mathbf{C}+\mathbf{Q}_{1}+\left(\rho_{1}^{-1}+\rho_{3}^{-1}\right) \mathbf{H}^{T} \mathbf{H} \\
& -\mathbf{P}_{1} \mathbf{N} \mathbf{A}_{d}\left(\left(\rho_{2}^{-1}+\rho_{4}^{-1}\right) \mathbf{H}_{d}^{T} \mathbf{H}_{d}-\mathbf{Q}_{1}\right)^{-1} \mathbf{A}_{d}^{T} \mathbf{N}^{T} \mathbf{P}_{1}, \\
\mathbf{J}_{12}= & \mathbf{P}_{1} \mathbf{B K}-\mathbf{K}^{T} \mathbf{R K}-\mathbf{F}^{T} \mathbf{P}_{2}+\gamma^{-2} \mathbf{P}_{1} \mathbf{N E} \mathbf{E}^{T} \mathbf{M}^{T} \mathbf{P}_{2} \\
& -\mathbf{P}_{1} \mathbf{N} \mathbf{A}_{d}\left(\left(\rho_{2}^{-1}+\rho_{4}^{-1}\right) \mathbf{H}_{d}^{T} \mathbf{H}_{d}-\mathbf{Q}_{1}\right)^{-1} \mathbf{A}_{d}^{T} \mathbf{M}^{T} \mathbf{P}_{2}, \\
\mathbf{J}_{22}= & (\mathbf{M A}-\mathbf{L} \mathbf{C}+\mathbf{F})^{T} \mathbf{P}_{2}+\mathbf{P}_{2}(\mathbf{M} \mathbf{A}-\mathbf{L} \mathbf{C}+\mathbf{F}) \\
& +\mathbf{P}_{2} \mathbf{M}\left(\gamma^{-2} \mathbf{E} \mathbf{E}^{T}+\rho_{3} \mathbf{D} \mathbf{D}^{T}+\rho_{4} \mathbf{D}_{d} \mathbf{D}_{d}^{T}\right) \mathbf{M}^{T} \mathbf{P}_{2} \\
& -\mathbf{P}_{2} \mathbf{M} \mathbf{A}_{d}\left(\left(\rho_{2}^{-1}+\rho_{4}^{-1}\right) \mathbf{H}_{d}^{T} \mathbf{H}_{d}-\mathbf{Q}_{1}\right)^{-1} \mathbf{A}_{d}^{T} \mathbf{M}^{T} \mathbf{P}_{2} \\
& +\mathbf{K}^{T} \mathbf{R K}+\mathbf{Q}_{2} .
\end{aligned}
$$

Design

$$
\begin{aligned}
\mathbf{K}= & \mathbf{R}^{-1} \mathbf{B}^{T} \mathbf{P}_{1}, \\
\mathbf{L}= & \lambda \mathbf{P}_{2}^{-1} \mathbf{C}^{T} / 2, \\
\mathbf{F}= & -\mathbf{M A}_{d}\left(\left(\rho_{2}^{-1}+\rho_{4}^{-1}\right) \mathbf{H}_{d}^{T} \mathbf{H}_{d}-\mathbf{Q}_{1}\right)^{-1} \mathbf{A}_{d}^{T} \mathbf{N}^{T} \mathbf{P}_{1} \\
& +\gamma^{-2} \mathbf{M E E}^{T} \mathbf{N}^{T} \mathbf{P}_{1},
\end{aligned}
$$

then substituting them into (23) can attain

$$
\begin{aligned}
\mathbf{J}_{11}= & (\mathbf{N A})^{T} \mathbf{P}_{1}+\mathbf{P}_{1}(\mathbf{N A})-\mathbf{P}_{1} \mathbf{B} \mathbf{R}^{-1} \mathbf{B}^{T} \mathbf{P}_{1}+\mathbf{C}^{T} \mathbf{C}+\mathbf{Q}_{1} \\
& +\mathbf{P}_{1} \mathbf{N}\left(\gamma^{-2} \mathbf{E} \mathbf{E}^{T}+\rho_{1} \mathbf{D} \mathbf{D}^{T}\right) \mathbf{N}^{T} \mathbf{P}_{1} \\
& +\rho_{2} \mathbf{P}_{1} \mathbf{N D}_{d} \mathbf{D}_{d}^{T} \mathbf{N}^{T} \mathbf{P}_{1}+\left(\rho_{1}^{-1}+\rho_{3}^{-1}\right) \mathbf{H}^{T} \mathbf{H} \\
& -\mathbf{P}_{1} \mathbf{N} \mathbf{A}_{d}\left(\left(\rho_{2}^{-1}+\rho_{4}^{-1}\right) \mathbf{H}_{d}^{T} \mathbf{H}_{d}-\mathbf{Q}_{1}\right)^{-1} \mathbf{A}_{d}^{T} \mathbf{N}^{T} \mathbf{P}_{1}, \\
\mathbf{J}_{12}= & \mathbf{0} \\
\mathbf{J}_{22}= & (\mathbf{M A}+\mathbf{F})^{T} \mathbf{P}_{2}+\mathbf{P}_{2}(\mathbf{M A}+\mathbf{F})+\mathbf{P}_{1} \mathbf{B} \mathbf{R}^{-1} \mathbf{B}^{T} \mathbf{P}_{1} \\
& -\lambda \mathbf{C}^{T} \mathbf{C}+\mathbf{P}_{2} \mathbf{M}\left(\gamma^{-2} \mathbf{E} \mathbf{E}^{T}+\rho_{3} \mathbf{D} \mathbf{D}^{T}\right) \mathbf{M}^{T} \mathbf{P}_{2} \\
& +\rho_{4} \mathbf{P}_{2} \mathbf{M D} \mathbf{D}_{d} \mathbf{D}_{d}^{T} \mathbf{M}^{T} \mathbf{P}_{2}+\mathbf{Q}_{2} \\
& -\mathbf{P}_{2} \mathbf{M} \mathbf{A}_{d}\left(\left(\rho_{2}^{-1}+\rho_{4}^{-1}\right) \mathbf{H}_{d}^{T} \mathbf{H}_{d}-\mathbf{Q}_{1}\right)^{-1} \mathbf{A}_{d}^{T} \mathbf{M}^{T} \mathbf{P}_{2} .
\end{aligned}
$$

Therefore, if there exists $\mathbf{P}_{1}>0$ and $\mathbf{P}_{2}>0$ such that $\mathbf{J}_{11}<0$ and $\mathbf{J}_{22}<0$, it implies to $\sup _{\mathbf{d} \in L_{2}} H[\mathbf{d}]<0$ and to guarantee the robust disturbance attenuation. The proof of this theorem is completed.

Remark 3: Generally, for any $\gamma$ for which a solution to (21) exists (which is used for the state feedback gain), we can find a $\lambda$ large enough such that a solution to the inequality (22) exists. It means that a high gain compensator can be used to accomplish the work. Besides, LMI technique [1] can be used to solve the two inequalities (21) and (22). Finally, we summarize the output feedback integral sliding mode controller:

$$
\begin{aligned}
\dot{\xi}(t)= & (\mathbf{M A}-\mathbf{L C}+\mathbf{F}) \xi(t)+(\Gamma-\mathbf{F} \mathbf{U}) \mathbf{y}(t), \\
\mathbf{s}(t)= & (\mathbf{G C B})^{-1} \mathbf{G}(\mathbf{y}(t)-\mathbf{y}(0)) \\
& +\int_{0}^{t} \mathbf{K}(\xi(q)-\mathbf{U} \mathbf{y}(q)) d q, \\
\mathbf{u}(t)= & -\mathbf{K}(\xi(t)-\mathbf{U} \mathbf{y}(t))-\kappa(t) \mathbf{s}(t) /\|\mathbf{s}(t)\| .
\end{aligned}
$$




\section{NUMERICAL EXAMPLES}

The following examples are simulated to verify the proposed controller design.

Example 1: Consider a nonlinear uncertain state-delay system [28] within the following form

$$
\begin{aligned}
\dot{\mathbf{x}}(t)= & \mathbf{A x}(t)+\mathbf{A}_{d} \mathbf{x}(t-\tau(t))+\mathbf{B} u(t) \\
& +\mathbf{B} f(t, \mathbf{x}(t), \mathbf{x}(t-\tau(t)))+\mathbf{d}(t, \mathbf{x}(t), \mathbf{x}(t-\tau(t))), \\
\mathbf{y}(t)= & \mathbf{C} \mathbf{x}(t),
\end{aligned}
$$

where $\mathbf{A}=\left[\begin{array}{ccc}-10 & 1 & 0 \\ 1 & 0 & 0 \\ 0 & 1 & -5\end{array}\right], \quad \mathbf{A}_{d}=\left[\begin{array}{ccc}0 & 0 & 0 \\ -1 & 0 & 1 \\ 0 & 0 & 0\end{array}\right], \quad \mathbf{B}=[0$ $-1 \quad 0]^{T}, \mathbf{C}=\left[\begin{array}{lll}0 & 0 & 1 \\ 0 & 1 & 0\end{array}\right]$, and delay time $\tau(t)=2+\sin t$.

The unknown matched disturbance is bounded as

$$
|f(\bullet)| \leq y_{2}^{4}(t) \sin ^{2} y_{1}(t-\tau(t)),
$$

where $\mathbf{y}(t)=\left[\begin{array}{ll}y_{1}(t) & y_{2}(t)\end{array}\right]^{T}$. The mismatched disturbance $\mathbf{d}(\bullet)$ is set as $\mathbf{d}(\bullet)=\left[\begin{array}{lll}d_{1}(\bullet) & 0 & d_{3}(\bullet)\end{array}\right]^{T}$ where

$$
\begin{aligned}
& d_{1}(\bullet)=\sqrt{2} \beta_{1}(t, \mathbf{x}(t), \mathbf{x}(t-\tau(t))) x_{1}(t) \\
& +\beta_{2}(t, \mathbf{x}(t), \mathbf{x}(t-\tau(t))) x_{1}(t-\tau(t)), \\
& d_{3}(\bullet)=\beta_{1}(t, \mathbf{x}(t), \mathbf{x}(t-\tau(t))) x_{3}(t) \\
& +\beta_{2}(t, \mathbf{x}(t), \mathbf{x}(t-\tau(t))) x_{3}(t-\tau(t)), \\
& \mathbf{x}(t)=\left[\begin{array}{lll}
x_{1}(t) & x_{2}(t) & x_{3}(t)
\end{array}\right]^{T}, \\
& \left|\beta_{1}(\bullet)\right| \leq y_{2}^{2}(t)\left|\sin y_{1}(t-\tau(t))\right|, \\
& \left|\beta_{2}(\bullet)\right| \leq\left|y_{1}(t-\tau(t))\right| \sin ^{2} y_{1}(t)+y_{2}^{2}(t) .
\end{aligned}
$$

Notice that $(\mathbf{A}, \mathbf{B}, \mathbf{C})$ has an invariant zero -10 and $\operatorname{rank}(\mathbf{C B})=1$. For solving algebraic Riccati inequalities (21) and (22) of this example, select parameters as $\gamma=0.53, \lambda=100, R=0.02, \mathbf{Q}_{1}=2 \mathbf{I}_{3}$, and $\mathbf{Q}_{2}=0.002 \mathbf{I}_{3}$.

Then solutions to (21) and (22) are

$$
\begin{aligned}
& \mathbf{P}_{1}=\left[\begin{array}{ccc}
0.1044 & 0.0048 & -0.0006 \\
0.0048 & 0.2727 & -0.0401 \\
-0.0006 & -0.0401 & 0.3776
\end{array}\right], \\
& \mathbf{P}_{2}=\left[\begin{array}{ccc}
3.8994 & -0.2369 & -1.4494 \\
-0.2369 & 9.8705 & -0.1304 \\
-1.4494 & -0.1304 & 3.6444
\end{array}\right] .
\end{aligned}
$$

Hence, we construct the full-order compensator as

$$
\begin{aligned}
\dot{\xi}(t)= & {\left[\begin{array}{ccc}
-9.6135 & 2.4036 & -9.8159 \\
0 & -5.0809 & -0.3574 \\
0.0297 & 3.2985 & -23.7214
\end{array}\right] \xi(t) } \\
& +\left[\begin{array}{cc}
25.4447 & 2.8451 \\
0.7149 & 0 \\
39.8163 & 3.6560
\end{array}\right] \mathbf{y}(t),
\end{aligned}
$$

$$
\begin{aligned}
v(t)= & {\left[\begin{array}{lll}
0.2408 & 13.6335 & -2.0045
\end{array}\right] \xi(t) } \\
& +\left[\begin{array}{ll}
1.7638 & 13.6335
\end{array}\right] \mathbf{y}(t)
\end{aligned}
$$

and design the sliding surface as

$$
s(t)=\left[\begin{array}{ll}
1 & -1
\end{array}\right](\mathbf{y}(t)-\mathbf{y}(0))-\int_{0}^{t} v(q) d q .
$$

Moreover, in order to avoid the chattering problem, the term $s(t) /|s(t)|$ is replaced with the saturation function, and the new version of controller is given by

$$
\begin{aligned}
u(t)= & v(t)-(1-\chi)^{-1}\left(\sigma_{1}+\sigma_{2}+\eta_{1}(t, \mathbf{y})\right. \\
& +\chi\|v(t)\|+\bar{\mu}) \operatorname{sat}(s(t), \varepsilon),
\end{aligned}
$$

where $\sigma_{1}=\sigma_{2}=3.75, \quad \eta_{1}(t, \mathbf{y})=y_{2}^{4}(t), \quad \chi=0, \quad \bar{\mu}=1$, and $\operatorname{sat}(\bullet)$ denotes the saturation function with $\varepsilon=0.002$. In comparison with [28], the controller in [28] was designed as

$$
u(t)=k(t, \mathbf{x}(t), \mathbf{x}(t-\tau(t))) \operatorname{sat}\left(y_{2}(t), \varepsilon\right),
$$

where

$$
\begin{aligned}
k(\bullet)= & 10.0499 \mu_{1}+\sqrt{y_{2}^{2}(t)+\left(y_{2}(t)-5 y_{1}(t)\right)^{2}} \\
& +\sqrt{2} y_{2}^{2}(t)\left|\sin y_{1}(t-\tau(t))\right|\left(\mu_{1}+\|\mathbf{y}(t)\|\right) \\
& +\eta_{2}+\left(\left|y_{1}(t-\tau(t))\right| \sin ^{2} y_{1}(t)+y_{2}^{2}(t)\right) \\
& \times\left(\mu_{2}+\|\mathbf{y}(t-\tau(t))\|\right)+y_{2}^{4}(t) \sin ^{2} y_{1}(t-\tau(t)) .
\end{aligned}
$$

The related parameters are designed as $\mu_{1}=\mu_{2}=2$, and $\eta_{2}=1$.

Figs. 1-5 show the comparison results using the initial state $\mathbf{x}(0)=\left[\begin{array}{lll}-2 & 2 & 3\end{array}\right]^{T}$ in the local regions (5) and $\xi(0)=\mathbf{0}$. The time responses of system states are shown in Figs. 1 to 3. All system states underlying these two methods converge to zero quickly. The comparison of responses of sliding function in the sliding layer is depicted in Fig. 4. For highlighting the difference we only chart sliding functions in one second. In our proposed method, the trajectory of sliding function indeed starts from zero and is bounded consistently in the sliding layer from the initial moment. The responses of the control inputs are given in the last figure. We also depict control forces in one second for highlighting the difference. The control input of our proposed method is smaller in the transient time and without chattering phenomena due to the replacement of saturation function. In contrast, our proposed control force in the transient time does not cause a high-speed switching behavior which exceeds the capability of most manipulators. Figs. 1-4 present that the integral sliding mode technique can suppress the effect of matched disturbances strongly. Although there exists the state-delay term and uncertainties in the nominal system, the system outputs asymptotically converge to zero. The simulation results demonstrate that the proposed controller design can guarantee the robust disturbance attenuation to outputs as the system in the sliding mode. 


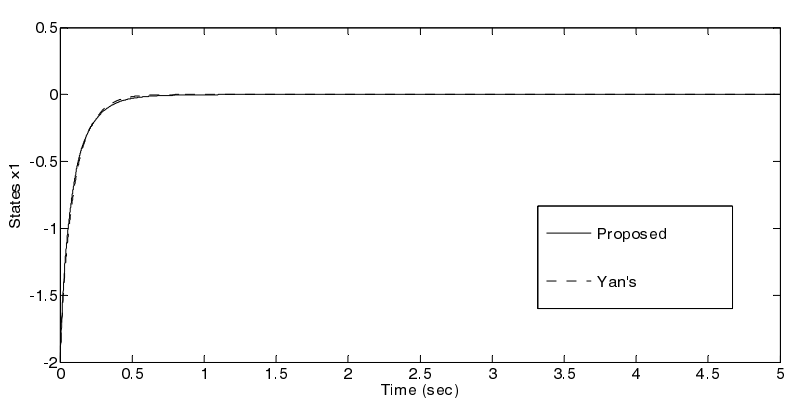

Fig. 1. The time responses of the state variable $x_{1}(t)$.

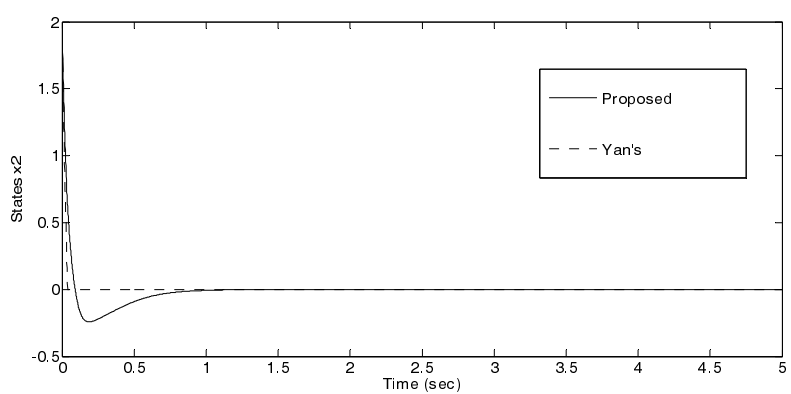

Fig. 2. The time responses of the state variable $x_{2}(t)$.

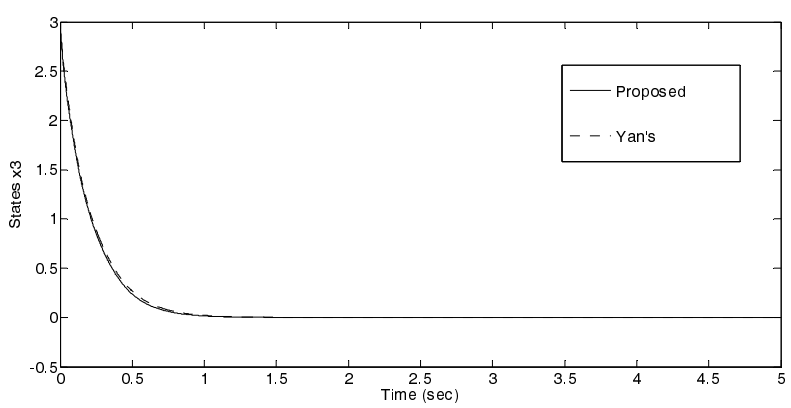

Fig. 3. The time responses of the state variable $x_{3}(t)$.

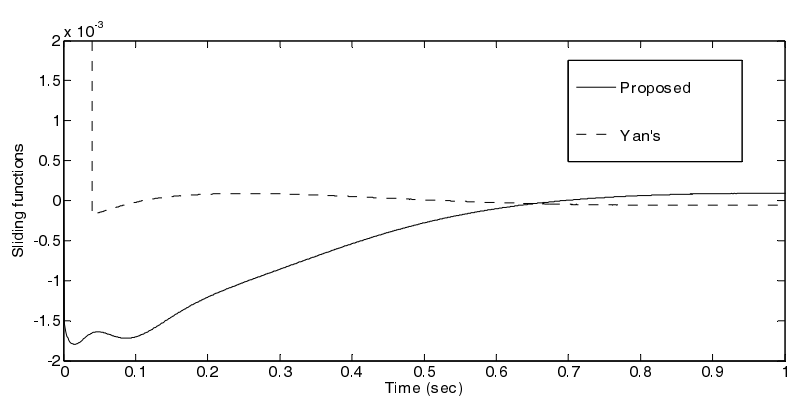

Fig. 4. The time responses of sliding function in the sliding layer.

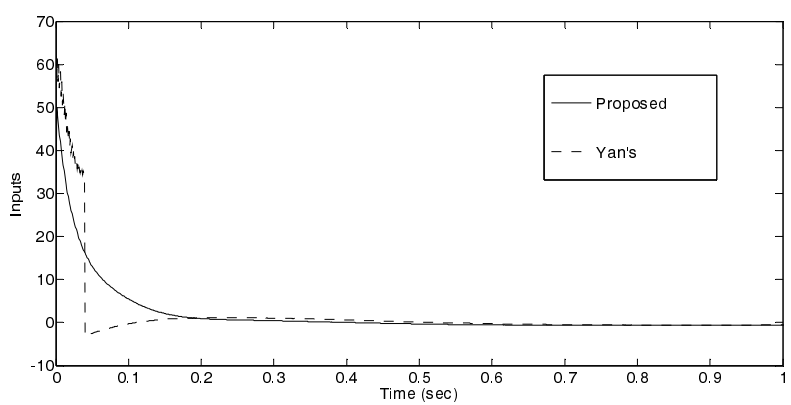

Fig. 5. The time responses of control signal.
Example 2: Consider an unstable example modified from the real example of chemical reactor system [6] within the corresponding form of system (2) with delay time $\tau=1$ as

$$
\begin{aligned}
\mathbf{A} & =\left[\begin{array}{cccc}
-4.93 & -1.01 & 0 & 0 \\
-3.20 & 0.3 & -12.8 & 0 \\
-6.40 & 0.347 & -32.5 & -1.04 \\
0 & 0.833 & 11.0 & -3.96
\end{array}\right], \quad \mathbf{B}=\left[\begin{array}{ll}
1 & 0 \\
0 & 1 \\
0 & 0 \\
0 & 0
\end{array}\right], \\
\mathbf{C} & =\left[\begin{array}{cccc}
1 & 0 & -0.2 & 0 \\
0 & 1 & 0 & 0.1
\end{array}\right], \quad \mathbf{E}=\left[\begin{array}{llll}
1 & 0 & 1 & 0
\end{array}\right]^{T},
\end{aligned}
$$

and $\mathbf{A}_{d}=\operatorname{diag}(1.92,1.92,1.87,0.724)$. Note that (A, B, C) has an unstable eigenvalue 0.4153 , invariant zeros 4.4463 and -33.377 , and $\operatorname{rank}(\mathbf{C B})=2$. The known parts of uncertainties in the system are given by

$$
\begin{aligned}
& \mathbf{D}=\left[\begin{array}{cccc}
-0.47 & 1.01 & 0 & 0 \\
-0.22 & -0.17 & 1.21 & 0 \\
0.63 & 0.347 & 0.91 & -1.04 \\
0 & 0 & 0.14 & -0.96
\end{array}\right], \\
& \mathbf{H}=\left[\begin{array}{cccc}
-0.55 & -0.02 & 0 & 0 \\
0.78 & -0.35 & 0 & 0 \\
0 & -0.72 & -0.49 & 0 \\
0 & 0.33 & -0.54 & -0.39
\end{array}\right], \\
& \mathbf{D}_{d}=\operatorname{diag}(0.47,0.26,-0.85,1.53), \\
& \mathbf{H}_{d}=\operatorname{diag}(-1.11,-0.21,1.26,0.47) .
\end{aligned}
$$

The external disturbances and unknown parts of uncertainties for system (2) are set as $\Phi(t)=r_{1}(t) \mathbf{I}_{4}$, $\Phi_{d}(t)=r_{2}(t) \mathbf{I}_{4}, d(t)=e^{-0.001 t} \sin 2 t$, and

$$
\mathbf{f}(\mathbf{x}, \mathbf{u}, t)=\left[\begin{array}{l}
0.12 u_{1} \sin t+0.08 u_{2} \cos 1.3 t+0.2 \sin x_{1} \\
0.07 u_{1} \cos 3 t+0.03 u_{2} \sin 5 t+0.3 \cos x_{2}
\end{array}\right],
$$

where $\mathbf{u}=\left[\begin{array}{ll}u_{1} & u_{2}\end{array}\right]^{T}, \quad r_{1}(t)$ and $r_{2}(t)$ are different random functions with values between -1 and 1 .

For solving algebraic Riccati inequalities (21) and (22) of this example, select parameters as $\gamma=0.53, \lambda=10$, $\mathbf{R}=0.02 \mathbf{I}_{2}, \mathbf{Q}_{1}=2 \mathbf{I}_{4}, \mathbf{Q}_{2}=0.002 \mathbf{I}_{4}, \rho_{1}=0.333, \rho_{2}=1$, $\rho_{3}=0.6$, and $\rho_{4}=5$ such that $\mathbf{Q}_{1}>\left(\rho_{2}^{-1}+\rho_{4}^{-1}\right) \mathbf{H}_{d}^{T} \mathbf{H}_{d}$. Then solutions to (21) and (22) are

$$
\begin{aligned}
& \mathbf{P}_{1}=\left[\begin{array}{cccc}
0.3493 & -0.0274 & -0.0731 & -0.0453 \\
-0.0274 & 0.3308 & 0.0096 & 0.0028 \\
-0.0731 & 0.0096 & 0.1228 & 0.1475 \\
-0.0453 & 0.0028 & 0.1475 & 0.3863
\end{array}\right], \\
& \mathbf{P}_{2}=\left[\begin{array}{cccc}
1.8870 & 0.0822 & 0.1077 & 0.4531 \\
0.0822 & 2.0885 & -0.0778 & 0.2438 \\
0.1077 & -0.0778 & 4.0664 & -0.3196 \\
0.4531 & 0.2438 & -0.3196 & 0.3283
\end{array}\right] .
\end{aligned}
$$


Hence, we construct the full-order compensator as

$$
\begin{aligned}
\dot{\xi}(t) & =\left[\begin{array}{rr}
-1.2852 & 0.0760 \\
0.0178 & -0.0706 \\
-6.4260 & 0.3802 \\
-0.1780 & 0.7062
\end{array}\right] \mathbf{y}(t) \\
& +\left[\begin{array}{cccc}
-5.8010 & 0.1534 & -5.4216 & 0.0241 \\
-0.6640 & -2.4837 & -1.0249 & -0.0067 \\
-5.4708 & 0.3417 & -31.8148 & 0.0781 \\
7.4909 & 0.8308 & 10.0792 & -2.3331
\end{array}\right] \xi(t),
\end{aligned}
$$

and design the sliding surface as

$$
\begin{aligned}
& \mathbf{s}(t)=\mathbf{y}(t)-\mathbf{y}(0)-\int_{0}^{t}\left[\begin{array}{cc}
-17.4651 & 1.3691 \\
1.3691 & -16.5404
\end{array}\right. \\
& \left.\begin{array}{cc}
3.6532 & 2.2646 \\
-0.4786 & -0.1376
\end{array}\right] \xi(q) d q \\
& -\int_{0}^{t}\left[\begin{array}{rr}
-17.4651 & 1.3691 \\
1.3691 & -16.5404
\end{array}\right] \mathbf{y}(q) d q .
\end{aligned}
$$

In order to avoid the chattering problem, the term $\mathbf{s}(t) /\|\mathbf{s}(t)\|$ in the controller (6) is replaced with the saturation function [27], and the new version of the controller is given by

$$
\begin{aligned}
\mathbf{u}(t)= & {\left[\begin{array}{rr}
-17.4651 & 1.3691 \\
1.3691 & -16.5404
\end{array}\right] \mathbf{y}(t)-(1-\chi)^{-1} } \\
& \times\left(\sigma_{1}+\sigma_{2}+\eta(t, \mathbf{y})+\chi\|\mathbf{v}(t)\|+\psi \bar{d}+\mu\right) \operatorname{sat}(\mathbf{s}(t), \varepsilon) \\
& +\left[\begin{array}{rrrr}
-17.4651 & 1.3691 & 3.6532 & 2.2646 \\
1.3691 & -16.5404 & -0.4786 & -0.1376
\end{array}\right] \xi(t),
\end{aligned}
$$

where $\sigma_{1}=\sigma_{2}=5, \eta(t, \mathbf{y})=2, \chi=\psi=0.8, \bar{d}=1, \mu=$ 2.5, and $\varepsilon=0.002$. Figs. $6-10$ chart the simulation results of the new version of controller using the initial state $\mathbf{x}(0)=\left[\begin{array}{llll}2 & 3 & 4 & 1\end{array}\right]^{T}$ and $\xi(0)=\mathbf{0}$. The time responses of system outputs are shown in Fig. 6. Figs. 7 and 8 show $\mathbf{s}(t)$ and $\|\mathbf{s}(t)\|$, respectively. In Fig. 8, the controlled system can maintain in the sliding layer the whole time. Fig. 9 depicts that the trajectories of $\mathbf{e}(t)$ are bounded around zero and do not converge to zero because of the mismatched disturbance. The responses of control inputs $\mathbf{u}(t)$ are given in Fig. 10. The replacement of the saturation function eliminates the chattering phenomenon. Although there exists an unstable root, a state-delay term, and a mismatched disturbance in the nominal system, the system outputs $\mathbf{y}(t)$ are finally bounded around zero in Fig. 6. The simulation results demonstrate that the proposed controller design can guarantee the robust disturbance attenuation to outputs $\mathbf{y}(t)$ once the system is in the sliding mode.

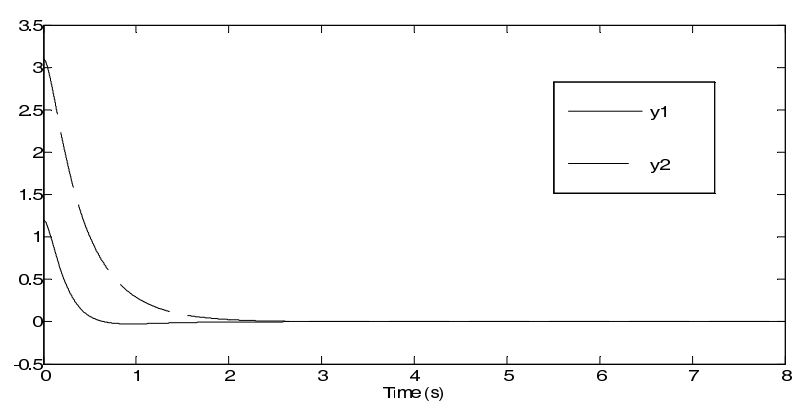

Fig. 6. System outputs.

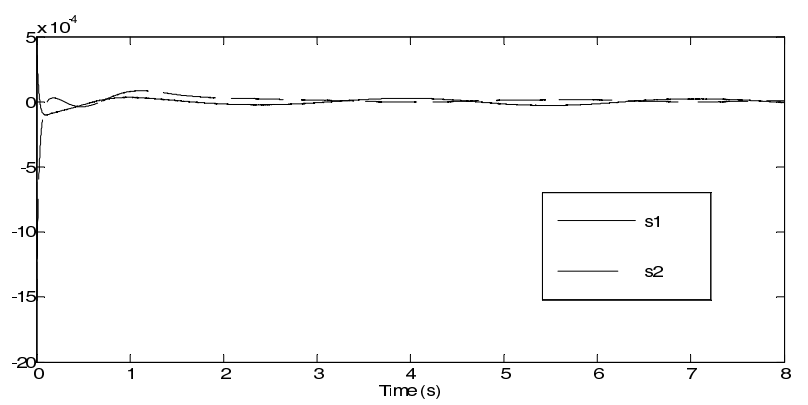

Fig. 7. Sliding surfaces.

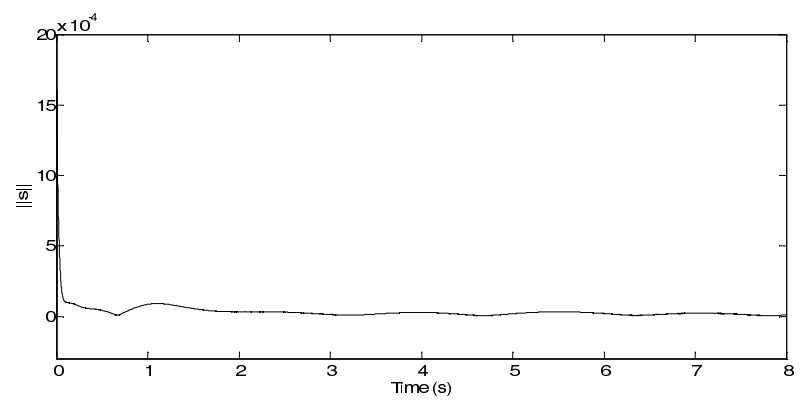

Fig. 8. Response of $\|\mathbf{s}(t)\|$.

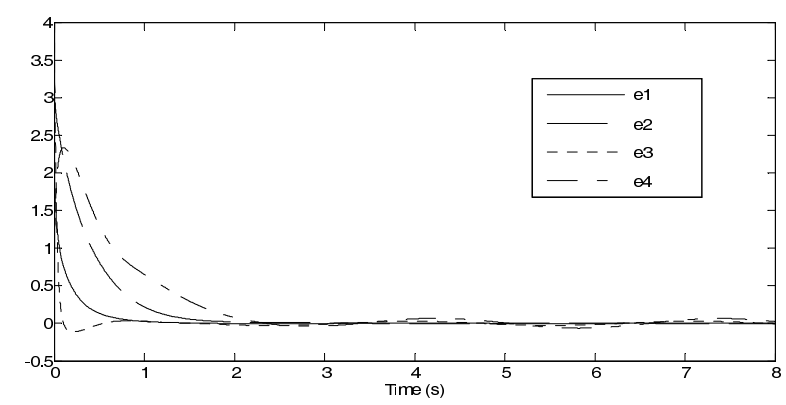

Fig. 9. Trajectories of $\mathbf{e}(t)$.

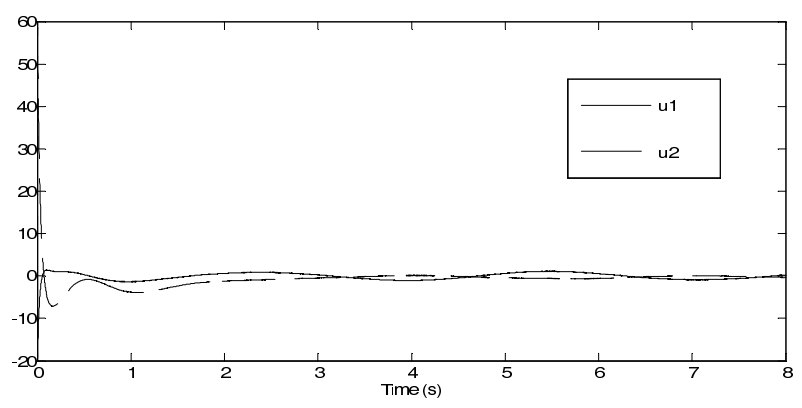

Fig. 10. System inputs. 


\section{CONCLUSIONS}

This paper has presented the output feedback integral sliding mode controller for a class of time-delay systems with structure uncertainties and mismatched disturbances. The auxiliary full-order compensator added into the design of the integral sliding surface can improve the synthesis problem of static output feedback sliding mode control. This paper utilizes the disturbance rejection condition in $H_{\infty}$ theory to derive two algebraic Riccati inequalities comprised of parameters of the system, controller, and compensator. When two algebraic Riccati inequalities have solutions, both the stability of the closed-loop system and the condition of robust disturbance attenuation can be guaranteed. Moreover, the designed controller can maintain that the system is always in the sliding mode from the initial moment. Finally, the simulation results of the real chemical reactor example demonstrated the feasibility of the propose control scheme.

\section{REFERENCES}

[1] Y. Xia and Y. Jia, "Robust sliding-mode control for uncertain time-delay systems: an LMI approach," IEEE Trans. on Automatic Control, vol. 48, no. 6, pp. 1086-1092, 2003.

[2] S. Qu and Y. Wang, "Robust control of uncertain time delay system: a novel sliding mode control design via LMI," Journal of Systems Engineering and Electronics, vol. 17, no. 3, pp. 624-628, 2006.

[3] Y. Niu, B. Chen, and X. Wang, "Sliding mode control for a class of nonlinear stochastic systems with state and input delays," International Journal of Control, Automation, and Systems, vol. 7, no. 3, pp. 365-370, 2009.

[4] C. C. Hua, Q. G. Wang, and X. P. Guan, "Memoryless state feedback controller design for time delay systems with matched uncertain nonlinearities," IEEE Trans. on Automatic Control, vol. 53, no. 3, pp. 801-807, 2003.

[5] J. H. Kim, "Delay-dependent robust and non-fragile guaranteed cost control for uncertain singular systems with time-varying state and input delays," International Journal of Control, Automation, and Systems, vol. 7, no. 3, pp. 357-364, 2009.

[6] Y. S. Lee, S. H. Han, and W. H. Kwon, "Receding horizon $H_{\infty}$ control for systems with a state-delay," Asian Journal of Control, vol. 8, no. 1, pp. 63-71, 2006.

[7] O. Santos and S. Mondié, "Guaranteed cost control of linear systems with distributed delays: a complete type functional approach," International Journal of Control, Automation, and Systems, vol. 8, no. 3, pp. 497-505, 2010.

[8] Z. Wang, L. Huang, Y. Zuo, and L. Zhang, " $H_{\infty}$ control for uncertain system with time-delay and nonlinear external disturbance via adaptive control method," International Journal of Control, Automation, and Systems, vol. 8, no. 2, pp. 266-271, 2010 .
[9] M. Chen and W. H. Chen, "Disturbance-observerbased robust control for time delay uncertain systems," International Journal of Control, Automation, and Systems, vol. 8, no. 2, pp. 445-453, 2010.

[10] A. Fattouh, O. Sename, and J. M. Dion, "An unknown input observer design for linear time-delay systems," Proc. of the 38th Conf. Decision and Control, Phoenix, Arizona, USA, pp. 4222-4227, 1999.

[11] Y. M. Fu, G. R. Duan, and S. M. Song, "Design of unknown input observer for linear time-delay systems," International Journal of Control, Automation, and Systems, vol. 2, no. 4, pp. 530-535, 2004.

[12] A. G. Wu, J. Dong, and G. R. Duan, "Robust Hinfinity estimation for linear time-delay systems: an improved LMI approach," International Journal of Control, Automation, and Systems, vol. 7, no. 4, pp. 668-673, 2009.

[13] M. Darouach, "Full order unknown inputs observers design for delay systems," Proc. of the 14th Mediterranean Conf. Control and Automation, pp. 1-5, 2006.

[14] M. Darouach, "Unknown inputs observers design for delay systems," Asian Journal of Control, vol. 9, no. 4, pp. 436-434, 2007.

[15] E. Fridman and U. Shaked, "A descriptor system approach to $H_{\infty}$ control of linear time-delay systems," IEEE Trans. on Automatic Control, vol. 47, no. 2, pp. 253-270, 2002.

[16] Y. Niu, J. Lam, X. Wang, and D. W. C. Ho, “Observer-based sliding mode control for nonlinear state-delayed systems," International Journal of Systems Science, vol. 35, no. 2, pp. 139-150, 2004.

[17] S. C. Bengea, X. Li, and R. A. DeCarlo, "Combined controller-observer design for uncertain time delay systems with application to engine idle speed control," Journal of Dynamic Systems, Measurement, and Control, vol. 126, pp. 772-780, 2004.

[18] J. W. Ko and P. G. Park, "Delay-dependent robust stabilization for systems with time-varying delays," International Journal of Control, Automation, and Systems, vol. 7, no. 5, pp. 711-722, 2009.

[19] X. G. Yan, S. K. Spurgeon, and C. Edwards, "Static output feedback sliding mode control for timevarying delay systems with time-delayed nonlinear disturbances," Proc. of the 17th IFAC World Congress, Seoul, Korea, pp. 8642-8647, 2008.

[20] X. R. Han, E. Fridman, S. K. Spurgeon, and C. Edwards, "On the design of sliding mode static output feedback controllers for systems with timevarying delay," Proc. of International Workshop Variable Structure Systems, pp. 136-140, 2008.

[21] M. C. Pai, "Observer-based adaptive sliding mode control for nonlinear uncertain state-delayed systems," International Journal of Control, Automation, and Systems, vol. 7, no. 4, pp. 536-544, 2009.

[22] L. Y. Wang and W. Zhan, "Robust disturbance attenuation with stability for linear systems with norm-bounded nonlinear uncertainties," IEEE Trans. on Automation Control, vol. 41, no. 6, pp. 
886-888, 1996

[23] L. Zhou, J. C. Doyle, and K. Glover, Robust and Optimal Control, Prentice Hall, NJ, 1996.

[24] G. Zhai, X. Chen, S. Takai, and K. Yasuda, "Stability and $H_{\infty}$ disturbance attenuation analysis for LTI control systems with controllers failures," Asian Journal of Control, vol. 6, no.1, pp. 104-111, 2004.

[25] C. Edwards and S. K. Spurgeon, Sliding Mode Control Theory and Application, Taylor \& Francis, London, 1998.

[26] F. Castanos and L. Fridman, "Analysis and design of integral sliding manifolds for systems with unmatched perturbations," IEEE Trans. on Automation Control, vol. 51, no. 5, pp. 853-858, 2006.

[27] J. J. E. Slotine and S. S. Sastry, "Tracking control of nonlinear systems using sliding surfaces with application to robot manipulators," International Journal of Control, vol. 38, no. 2, pp. 465-492, 1983.

[28] X. G. Yan, S. K. Spurgeon, and C. Edwards, "Static output feedback sliding mode control for timevarying delay systems with time-delayed nonlinear disturbances," International Journal of Robust Nonlinear Control, vol. 20, pp. 777-788, 2010.

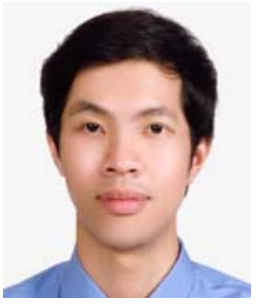

Huan-Chan Ting received his B.S. degree in Electrical Engineering from National Taipei University of Technology, Taipei, Taiwan in 2003, and his Ph.D. degree in Electrical and Control Engineering from National Chiao-Tung University, Hsinchu, Taiwan in 2011. His major fields of research are sliding mode control theorem, robust control, and power electronics.

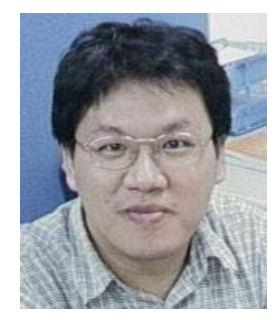

Jeang-Lin Chang received his B.S., M.S., and Ph.D. degrees in Electrical and Control Engineering from National Chiao-Tung University, Hsinchu, Taiwan. $\mathrm{He}$ is a professor in the Department of Electrical Engineering, Orient Institute of Technology. His researches include variable structure control, robust control, and discrete signal processing.

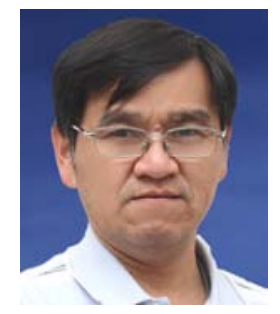

Yon-Ping Chen received his B.S. degree in Electrical Engineering from National Taiwan University in 1981, and his M.S. and Ph.D. degrees in Electrical Engineering from University of Texas at Arlington, U.S.A. He is a professor in the Institute of Electrical Control Engineering, National Chiao-Tung University. His researches include variable structure control, intelligent control, and image processing. 